\title{
L'installation et la mise en æuvre de barrages gonflables \\ permettant la surélévation de barrages existants
}

\author{
A. Dillon, Bridgestone Industrial Ltd
}

M. Carnobroda, MCT sarl

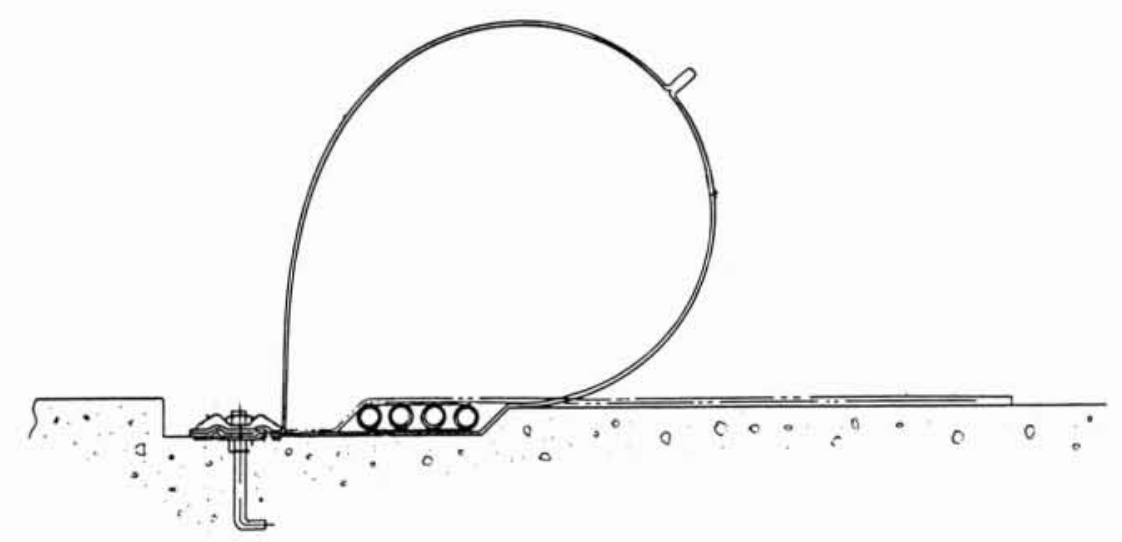

1. Coupe d'un barrage gonflable type (rempli d'air).

\section{Introduction}

L'augmentation de la capacité de stockage d'une retenue ou de la hauteur de chute d'eau permet d'améliorer le potentiel des barrages existants. Les dispositifs qui permettaient antérieurement de réaliser une telle surélévation étaient soit les hausses de déversoir, dont la hauteur est limitée et dont la mise en œuvre est peu flexible, soit divers types de vannes mécaniques dont la technique n'est pas toujours simple et dont l'installation sur la crête de barrages existants s'avère parfois peu rentable. Le barrage gonflable est une nouvelle alternative qui offre une grande souplesse d'opération pour un coût modéré.

La première partie de ce document présente le concept du barrage gonflable. La deuxième partie donne un aperçu de la procédure d'installation d'un barrage gonflable sur la crête d'un barrage existant. Et pour conclure, dans la troisième partie, nous donnerons quelques exemples de la mise en cuvre de barrages gonflables pour des projets hydroélectriques.

\section{Description du barrage gonflable}

Un barrage gonflable est une enveloppe de caoutchouc étanche fixée à une fondation. Lorsqu'on la remplit d'air, l'enveloppe se relève, créant ainsi une dérivation du cours d'eau ou surélevant la crête du barrage. Lorsqu'on ouvre un robinet de purge, l'air s'évacue et l'enveloppe se remet à plat, permettant à l'eau de s'écouler librement par dessus. Le mot «barrage gonflable " n'est qu'un des termes désignant un tel dispositif. On dit également "barrage en caoutchouc ", "vanne gonflable ", " seuil gonflable ", et " fabridam ».

Les barrages gonflables sont des structures simples. L'élément le plus important est le corps du barrage, une enveloppe habituellement constituée d'un caoutchouc armé de nylon qui est ancré à une fondation dans un cours d'eau ou sur la crête d'un barrage. Par l'intermédiaire d'une tuyauterie connectée à l'enveloppe, un groupe motocompresseur remplit d'air le corps en caoutchouc qui se gonfle. D'autres tuyauteries à l'intérieur de l'enveloppe permettent à l'air de s'évacuer librement pendant le dégonflage. Dans la figure 1 ci-dessus on voit une nervure qui est une caractéristique de certains types de barrages gonflables. Cette nervure a deux fonctions : d'abord, elle minimise les oscillations du barrage quand la charge de la nappe déversante est forte; et deuxièmement, elle crée un bord aval plat lorsque le corps du barrage est dégonflé. Ce dernier point est important, car sans la nervure le bord aval de l'enveloppe prendrait la forme d'une "bosse " susceptible d'être rapidement usée et endommagée par les vibrations et le charriage. 
L'enveloppe en caoutchouc d'un barrage gonflable doit être solidement fixée à sa fondation. Les fixations en acier que l'on voit dans la figure $l$ sont installées tout le long du bord amont du corps du barrage gonflable afin de le fixer, ou l'ancrer sur place. Elles comprennent des éléments encastrés qui forment une partie de la fondation, et des plaques supérieures de bridage. Ces plaques de bridage sont fixées avec des écrous, et servent non seulement à ancrer le corps du barrage à sa fondation mais aussi à créer une fermeture étanche entre les parties supérieure et inférieure du corps en caoutchouc, c'est-à-dire lui donner sa forme de boudin.

\section{Caractéristiques des barrages gonflables}

Les barrages gonflables présentent plusieurs avantages qui sont le résultat de leur conception originale, par exemple : une longue portée, un nombre réduit de piles, un angle de pente latérale libre, des fondations simples, une maintenance réduite, le passage des glaces.

- Une longue portée: Puisqu'un barrage gonflable est ancré tout le long de sa fondation, les contraintes ne sont pas concentrées à des endroits tels que glissières ou axes dans les piles; elles sont distribuées sur toute la longueur de la fondation, ce qui permet l'adoption de portées longues. Des barrages en caoutchouc avec des portées de plus de $100 \mathrm{~m}$ ont été installés.

- Un nombre réduit de piles : Les vannes gonflables nécessitent peu de piles puisque les portées sont longues.

- Angle de pente latérale libre: L'inclinaison de la pente latérale est libre, car les vannes gonflables n'ont pas besoin de se plaquer contre des murs guideaux ou des piles à angle droit.

- Des fondations simples: L'installation d'un barrage gonflable sur une fondation existante est relativement simple. D'ailleurs, il n'y a pas besoin de structures aériennes, d'installations complexes dans les fondations, ou d'une grande chambre de contrôle.

- La maintenance: Il n'y a nul besoin de peinture, les besoins en maintenance sont réduits puisque le caoutchouc est un matériau qui ne se corrode pas, et le barrage gonflable ne comporte pas de système mécanique situé sous l'eau.

- Le passage des glaces: Le gel ne peut bloquer un barrage gonflable en position gonflée. La flexibilité d'un barrage gonflable lui permet aussi de s'effacer légèrement lorsque la glace exerce une certaine pression, ce qui permet à la glace de franchir le corps en caoutchouc qui reprend aussitôt sa forme initiale.

\section{Comparaison de barrages gonflables remplis d'air et d'eau}

Les premiers barrages gonflables employaient exclusivement de l'eau comme moyen de remplissage en raison de sa masse anti-oscillatoire. Le développement de mesures anti-oscillatoires spécifiques, notamment la nervure sur le corps du barrage, a entraîné un recentrage vers les barrages gonflés à l'air. Ceci constitue un développement très positif, car l'air à plusieurs avantages par rapport à l'eau, notamment :

- La facilité du contrôle : L'emploi de l'air permet l'utilisation d'un simple groupe moto-compresseur, d'une seule ouverture de remplissage/purge dans l'enveloppe, et d'une tuyauterie de petit diamètre. L'utilisation de l'eau nécessite une pompe, des tuyauteries de grand diamètre, et plusieurs ouvertures à la base du barrage à connecter à des conduites de vidange pour l'évacuation de l'eau de remplissage.

- L'installation: Les équipements simples nécessaires pour un barrage gonflé à l'air permettent une installation relativement rapide.

- La maintenance: Les tuyauteries d'eau peuvent se corroder ou se trouver bouchées par les sédiments.

- Le temps du gonflage/dégonflage: Bien que le temps de gonflage et dégonflage soit fonction des dimensions de l'enveloppe, de la puissance du compresseur ou de la pompe, et du diamètre des tuyauteries, en pratique le gonflage ou dégonflage d'un barrage gonflable rempli d'air prend en général une dixième du temps nécessaire pour un barrage gonflé à l'eau.

- La qualité de l'eau: L'eau laissée dans l'enveloppe pendant une période prolongée peut se polluer et contaminer l'eau aval lors du dégonflage.

\section{Systèmes de mancuvre}

L'existence d'une large gamme de systèmes de contrôle permet l'adaptation du barrage gonflable à pratiquement toutes les applications. La méthode de contrôle la plus simple utilise un compresseur d'air standard, un manomètre, et un robinet de gonflage/dégonflage. Un système mécanique de dégonflage automatique assure la protection contre les crues par ouverture du robinet de dégonflage dès que le niveau d'eau amont atteint un point pré-réglé. Des systèmes plus sophistiqués utilisent un capteur de pression à l'intérieur de l'enveloppe, un capteur de niveau à l'amont, des moto-vannes, et un ordinateur dont le fonctionnement intégré règle le débit d'eau déversant par dessus le barrage en effectuant de faibles modifications de la pression d'air. Ce système maintient le niveau amont afin qu'un débit constant passe dans les conduits. Entre ces deux extrêmes il existe des systèmes de contrôle qui surveillent la pression interne du corps du barrage et déclenchent le démarrage du compresseur dès qu'une chute de pression est détectée. Il est également possible d'assurer la commande à distance et l'interface du barrage gonflable avec des systèmes de contrôle hydroélectrique ainsi que le contrôle du débit général du réseau hydraulique.

\section{Développement du concept du barrage en caoutchouc}

Les barrages en caoutchouc existent depuis 30 ans. Ils ont été conçus en 1956 par le Los Angeles County Flood Control District en remplacement des hausses de déversoir dont le retrait s'était souvent avéré impossible avant le 
passage de crues soudaines qui inondaient donc à l'aval et/ou arrachaient le dispositif à hausses au passage. Bien qu'ils constituaient une nouvelle approche au problème du contrôle des eaux, les premiers barrages gonflables rencontraient des problèmes de deux ordres: les déficiences des caoutchoucs employés pour leur fabrication, et des problèmes associés à l'utilisation de l'eau comme moyen de gonflage.

Les premiers barrages gonflables étaient du type à remplissage d'eau. L'eau était employée puisque la masse ou "ballast " qu'elle fournissait était nécessaire pour contrer l'oscillation du corps en caoutchouc. Les problèmes associés à l'utilisation d'eau ont déjà été décrits. Un problème supplémentaire rencontré en Californie était le besoin de maintenir en permanence un réservoir plein d'eau pour le remplissage du barrage. L'air, en revanche, est non seulement disponible à volonté et gratuitement, mais n'a pas non plus besoin d'être filtré. L'adoption du gonflage à l'air a récemment permis d'installer des barrages gonflables dans des sites aux conditions climatiques très froides où l'eau dans les tuyauteries, voire même dans l'enveloppe en caoutchouc, pourrait geler.

Les premiers barrages gonflables ont donné des résultats mitigés. Quelques barrages encore en fonctionnement ont plus de vingt ans tandis que d'autres étaient hors d'usage quelques années après l'installation. Leur défaillance était souvent due à l'usure du corps en caoutchouc. Il est intéressant de noter qu'il se dégradait davantage lorsqu'il était dégonflé que lorsqu'il était relevé. Ceci est dû au fait que les premiers barrages ne reposaient pas à plat mais avaient une "bosse" lorsqu'ils reposaient sur la fondation, cette bosse subissant la fatigue engendrée par l'écoulement de l'eau, et étant endommagée par les charges charriées au fond de l'eau. Des améliorations de la conception ont entraîné l'utilisation d'un caoutchouc plus résistant qui ne présente pas de bosse au repos, ainsi que l'utilisation de l'air plutôt que de l'eau. Toutes ces améliorations donnent aux barrages gonflables modernes une durée de vie estimée à plus de trente ans. La fiabilité des barrages gonflables n'est plus en question, puisque plus de 1500 de ces systèmes existent dans le monde. On cherche maintenant à développer au maximum le potentiel de ce concept.

\section{Les applications}

Bien que le barrage gonflable ait fait sa première apparition aux Etats-Unis, ce sont les Japonais qui ont le mieux accepté l'idée et fait avancer cette technologie. Au Japon la plupart de ces barrages se trouvent dans le lit de rivières où ils sont relevés au printemps afin de dévier les énormes volumes d'eau nécessaires pour l'inondation des rizières. En dehors de l'irrigation, les barrages gonflables sont employés pour recharger la nappe phréatique (percolation de l'eau dans l'aquifère), fonction pour laquelle ils étaient initialement conçus en Californie, où existe une dizaine de barrages assurant ce rôle. L'absence de risque de corrosion de l'enveloppe en caoutchouc d'un barrage gonflable a incité en outre une application en tant que barrière contre les marées et pour le contrôle des cours d'eau contaminés.
Le système de barrages gonflables le plus long du monde mesure 600 mètres et comporte 7 portées. Il se trouve en Pennsylvanie, aux Etats-Unis, où, du printemps jusqu'à l'automne, il convertit une rivière en lac de loisirs. Parce que le barrage repose à plat quand il ne sert pas et comporte peu de piles, il permet une excellente évacuation des sédiments en hiver lorsque l'aire de loisirs n'est plus utilisée et que le barrage est dégonflé. Récemment le barrage gonflable a été utilisé pour surélever la crête de barrages afin d'augmenter la chute d'eau pour la production hydroélectrique, ou d'augmenter la capacité de retenues. Dans une adaptation de cette application, les barrages gonflables ont été modifiés afin de coiffer les barrages voûtes où ils peuvent s'adapter à la courbure de la voûte. Trois exemples de cette application de surélévation seront présentés, ainsi que l'exemple d'une vanne gonflable installée lors de la réhabilitation du barrage de prise d'un aménagement hydroélectrique. Mais nous allons présenter d'abord le processus d'installation d'un barrage gonflable.

\section{Installation du barrage gonflable}

\section{Introduction}

L'élément principal à prendre en compte dans la description de l'installation d'un barrage gonflable est le type de fondation, c'est-à-dire savoir s'il est installé sur une fondation neuve ou s'il est rapporté sur la crête d'un barrage existant. Etant donné que nous parlons ici de la réhabilitation des barrages, cette présentation traitera uniquement de la marche à suivre dans ce dernier cas. L'autre facteur important dans l'installation d'un barrage gonflable - la fixation de l'enveloppe en caoutchouc - reste identique dans les deux cas.

\section{Installation de la fondation}

La mise en place des tuyauteries et des éléments encastrés nécessaires pour les barrages gonflables implique des techniques conventionnelles de percement et de scellement dans le béton existant. Cette méthode habituelle qui est décrite ci-après est suivie de l'explication d'une méthode alternative qui s'emploie quand la crête du barrage n'est pas assez large pour supporter le barrage gonflable.

1. Une tuyauterie est installée pour connecter le compresseur d'air à la portée du barrage gonflable (fig. 2, page suiv.).

2. Une tuyauterie de niveau d'eau est posée dans la fondation à l'amont du barrage gonflable. Cette tuyauterie permet de transférer le niveau de l'eau amont dans une chambre sous la salle de contrôle afin d'activer le système de dégonflage automatique (fig. 2).

3. Une tuyauterie est posée dans la fondation. Cette tuyauterie se décharge sur le côté à l'aval du barrage et sert à drainer l'eau de condensation de l'intérieur de l'enveloppe (fig. 2).

4. Des trous sont percés dans la fondation, espacés normalement de $200 \mathrm{~mm}$, qui recevront les tiges d'ancrage. 


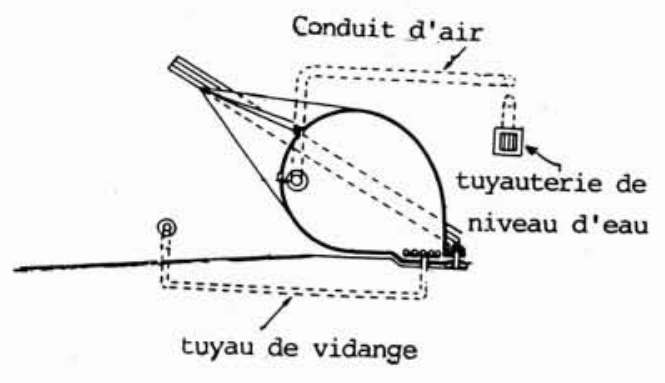

VUE VERS LA PENTE LATERALE

2. Connexion de la tuyauterie.

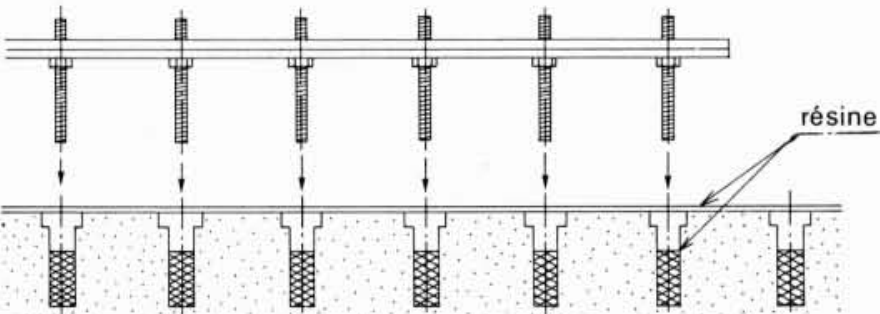

3. Ensembles de plaques encastrées/boulons d'ancrage.

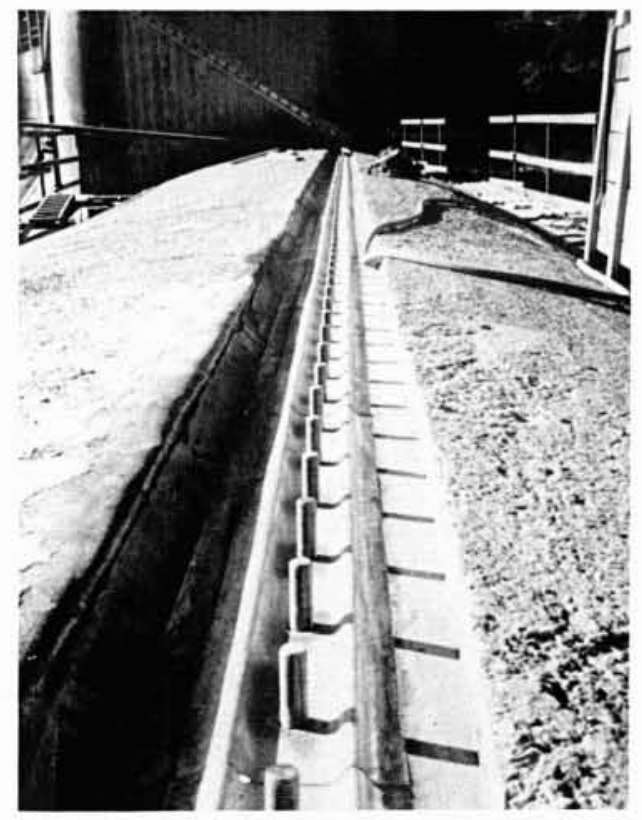

4. Travaux de fondation finis.

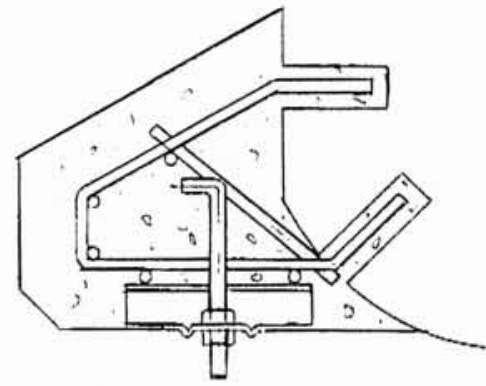

5. Coupe de lancrage sur le parement amont de la crète.

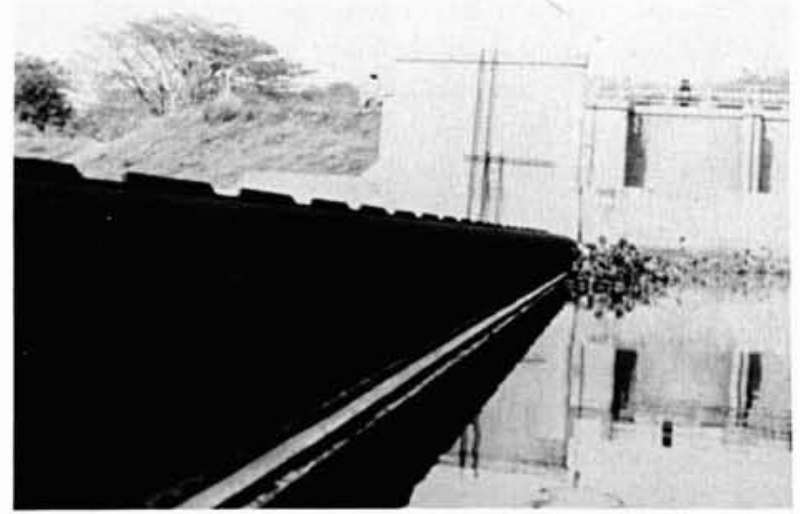

6. Détail du système d'ancrage du barrage de Non Wai.

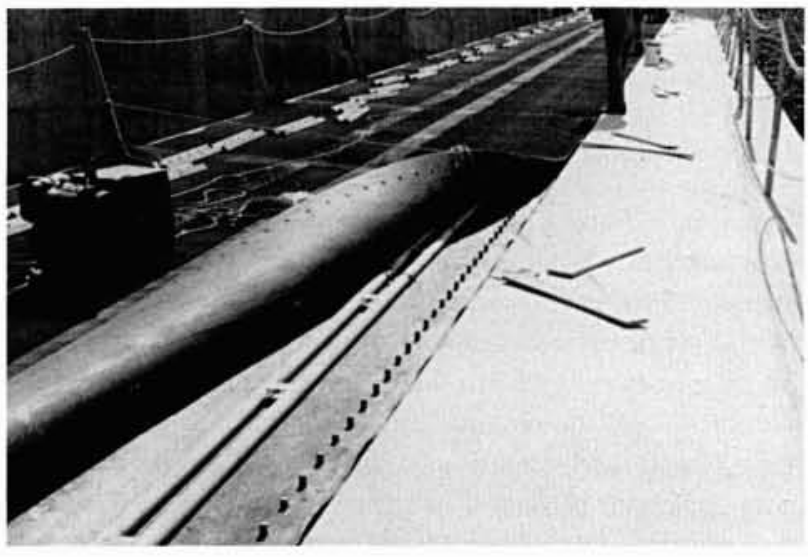

7. Corps du barrage gonflable replié. 
5. De la résine époxyde est placée dans les trous d'ancrage. 6. Une série d'ensembles d'environ 6 tiges d'ancrage avec leur plaque de bridage est posée en continu dans les trous d'ancrage sur toute la longueur de la fondation (fig. 3). L'ensemble des travaux de fondation prêt à recevoir l'enveloppe du barrage gonflable est montré dans la figure 4 .

\section{Fondation alternative}

Lorsque la place sur la crête d'un barrage est insuffisante pour l'installation des éléments d'ancrage encastrés d'une vanne gonflable et permettre à la vanne de se poser correctement à plat une fois qu'elle est dégonflée, une extension en porte à faux est ajoutée au parement amont de la crête. Les ensembles des plaques encastrées et les armatures en acier y sont ancrés (fig. 5),

Cette méthode a été employée pour le barrage gonflable de Non Wai en Thailande. Avec une longueur de $125 \mathrm{~m}$, c'est la portée de vanne gonflable la plus longue du monde. Bien que ce barrage ait une hauteur de seulement $60 \mathrm{~cm}$, du fait de la faible pente de la rivière il augmente de façon considérable la capacité de stockage. La figure 6 illustre le barrage gonflable de Non Wai avec une retenue encore sous le niveau de la crête du barrage en béton.

\section{Installation de l'enveloppe du barrage gonflable}

L'enveloppe du barrage gonflable est livrée enroulée sur un dévidoir. Chaque portée est livrée complète: il n'y a nul besoin de travaux de recouvrement et de thermo-soudage sur le chantier. Les procédures de fixation du corps en caoutchouc et des tuyauteries associées se déroulent sommairement comme suit :
1. L'enveloppe est déroulée sur la fondation. Les perforations le long de son bord amont sont passées sur les tiges d'ancrage scellées dans la fondation.

2. La partie supérieure du corps en caoutchouc est repliée en arrière afin d'exposer l'intérieur (fig. 7).

3. Les tuyauteries de purge d'air sont placées à l'intérieur du corps du barrage (fig. 7).

4. Des ouvertures sont découpées dans la partie latérale du corps du barrage en caoutchouc, pour la connexion de la tuyauterie d'air et à la base pour la connexion de la tuyauterie de vidange d'eau de condensation. Des collets sont passés par les ouvertures et insérés dans les tuyauteries.

5. La partie supérieure du corps en caoutchouc qui était repliée à l'étape 2 est remise en place, les trous mis en place sur les tiges d'ancrage.

6. Les plaques de bridage sont mises en place sur les tiges d'ancrage et serrées par boulonnage (fig. 8).

\section{Exemples de réalisations}

\section{Introduction}

Un certain nombre de barrages gonflables ont été installés sur la crête de barrages: la chute d'eau supplémentaire qu'ils créent pour l'industrie hydroélectrique, ainsi que l'optimisation de fonctionnement qui résulte du contrôle par simple effleurement de boutons-poussoirs, permettent une augmentation de la production d'énergie et un remboursement rapide des investissements.

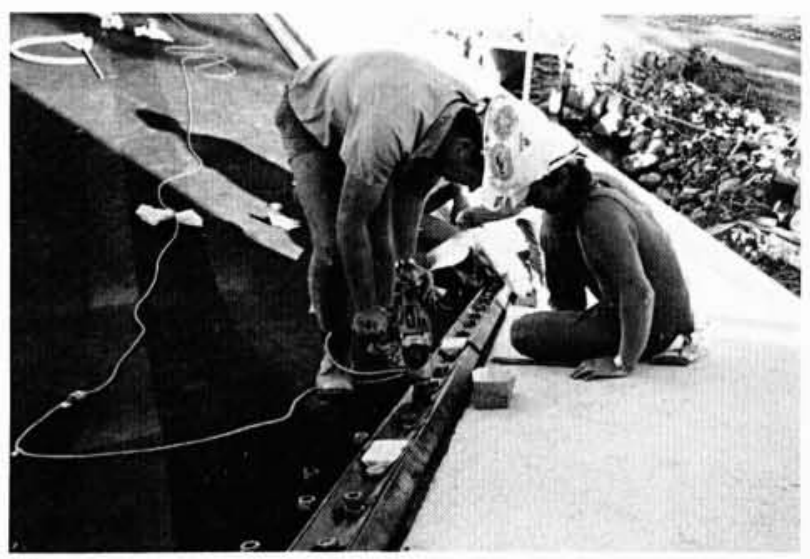

8. Les plaques de bridage ancrent l'enveloppe sur la fondation. 


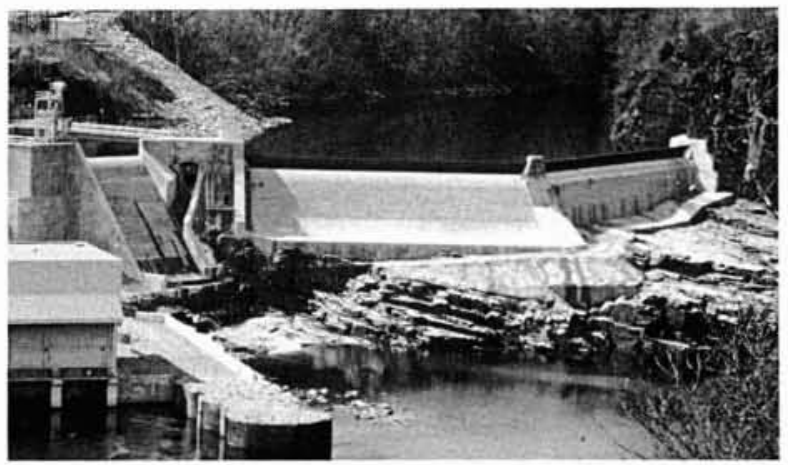

Le barrage de Palmer Falls avec barrage gonflable en tête.

La crête du barrage avant et après.
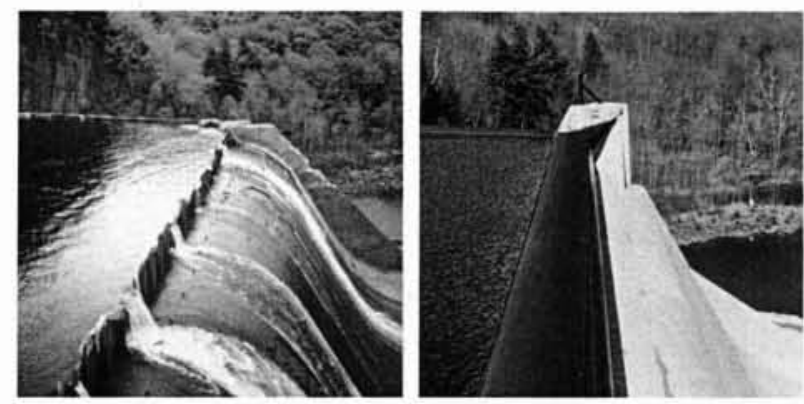

\section{Palmer Falls}

Un barrage en caoutchouc à deux portées fut installé sur la crête du barrage en béton de $26 \mathrm{~m}$ de haut de Palmer Falls sur le Hudson River dans l'état de New York, EtatsUnis, lors de la réhabilitation en 1987 de ce barrage vieux de 70 ans. Les deux portées ont la même hauteur de $1,83 \mathrm{~m}$; l'une mesure $61,61 \mathrm{~m}$ de long, l'autre $45,48 \mathrm{~m}$. Le barrage est exploité par le Maître de l'Ouvrage, International Paper Company, qui se sert de l'énergie produite pour sa papeterie et vend l'excédent au réseau local.

Les barrages en caoutchouc de Palmer Falls remplacent des hausses de déversoir. En termes opérationnels, ils présentent une amélioration importante, car ils bénéficient de la facilité de fonctionnement de clapets grâce au contrôle simple par le moyen de boutons-poussoirs. Ceci supprime le risque de perdre une hauteur de chute d'eau supplémentaire en attendant la remise en place des hausses une fois qui ont été retirées, tout en supprimant les risques courus par le personnel travaillant sur la crête. De plus, les barrages gonflables sont plus hauts que la hauteur maximale admise pour les hausses de déversoir. En ajoutant à ces avantages une installation relativement simple - qui s'effectue pendant la réhabilitation du barrage en béton lui-même - le retour sur l'investissement et la rentabilité du projet sont assurés très rapidement.

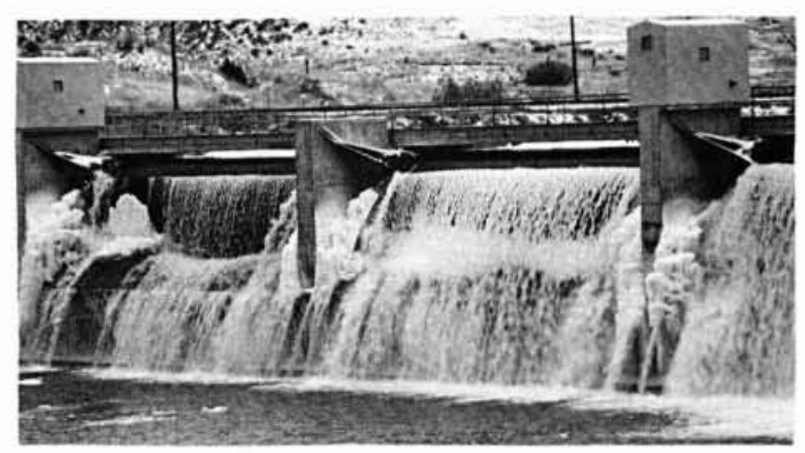

La glace ne peut bloquer les barrages gonflables en position relevée.

Sept portées de barrages gonflables.

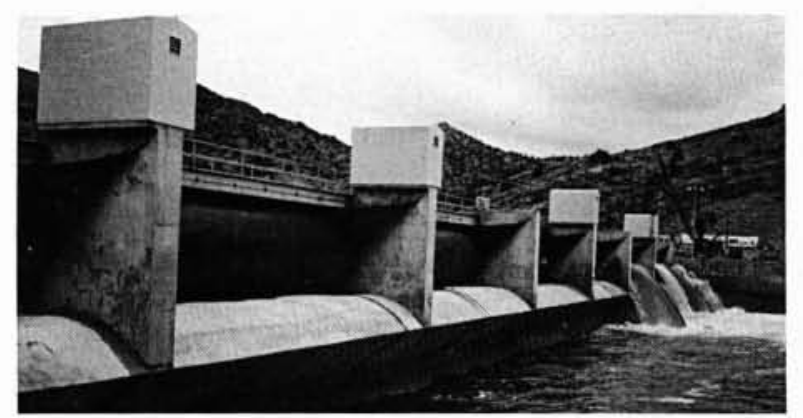

\section{Broadwater}

Sujet à des températures hivernales en dessous de $-40^{\circ} \mathrm{C}$, le dispositif de barrages gonflables de Broadwater, installé en 1989 dans le Montana aux Etats-Unis, fonctionne sous les températures les plus froides subies à ce jour par un barrage gonflable.

Le barrage de Broadwater constitue le seuil de prise d'un aménagement hydroélectrique. Comme à l'origine le barrage de base fut conçu pour des vannes de déversoir en bois, la distance entre les piles était limitée.

Bien que deux ou trois portées de barrages gonflables auraient pu suffire pour la totalité de la crête du barrage, sept portées ont été effectivement mises en œuvre, chacune de $3,35 \mathrm{~m}$ de haut et $15,54 \mathrm{~m}$ de long, afin de s'adapter à la structure existante et de réduire les modifications de cette structure. 


\section{Hakone}

Le barrage caoutchouc de Hakone est un exemple d'amélioration de vannes. Cet aménagement hydroélectrique au fil de l'eau dans une région montagneuse de loisirs à l'ouest de Tokyo, au Japon, a une capacité de production de 1,5 MW en turbinant l'eau déviée par le barrage gonflable de $1,80 \mathrm{~m}$ de haut sur $14 \mathrm{~m}$ de long installé sur l'ancienne fondation d'une vanne secteur. Le barrage gonflable fut installé après les dégâts subis par la vanne secteur en raison des craintes que l'ancienne vanne ne soit plus en mesure de se relever en toute fiabilité pendant les crues. Lorsqu'il est dégonflé pendant le passage des typhons, le barrage gonflable reste à plat sur sa fondation sous une nappe d'eau d'une hauteur maximale de $4 \mathrm{~m}$. Dans ce torrent d'une pente de $1 / 60$, le fait que la vanne gonflable s'efface complètement permet aux troncs d'arbres et aux blocs rocheux de passer sans encombre. Il a été nécessaire d'installer un tapis de caoutchouc spécial (apron rubber) sur la fondation en béton afin de la protéger de l'abrasion des blocs charriés.

En raison de l'importance de la population habitant à l'aval du seuil, il était primordial que la conception de la vanne assure une totale protection contre les crues. Les défis à relever pour une telle installation en haute montagne, avec des torrents à pentes très raides, des crues brutales et une capacité de stockage dans le lit limitée, ont pour conséquence que les systèmes à vannes comportent un risque potentiel pour les zones à l'aval. Le barrage gonflable permettant de sélectionner le dégonflage automatique garantit l'évacuation optimale des eaux de crues.

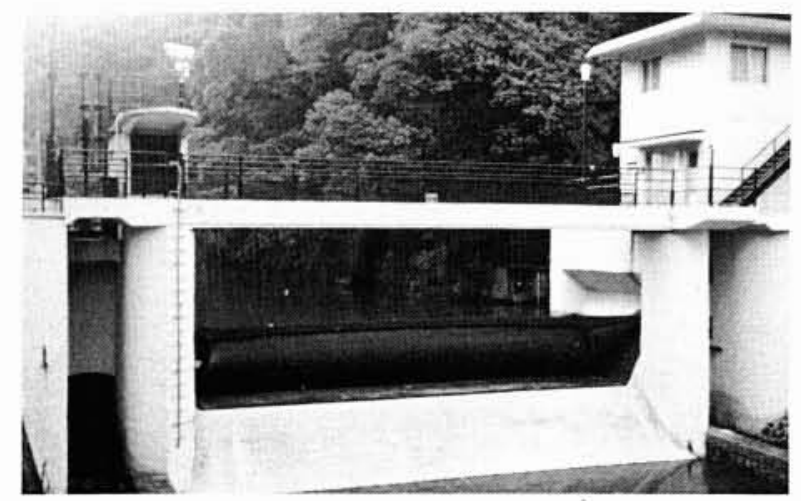

Le barrage gonflable.

La vanne secteur avant son remplacement.

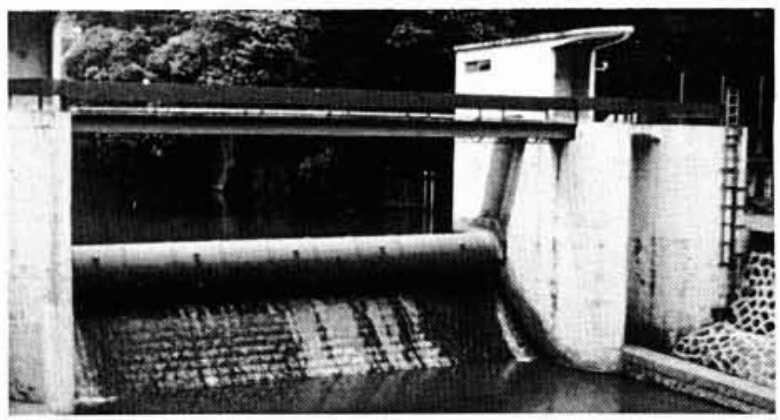

\section{Pit Three}

En 1989 le barrage de Pit Three a été la première installation dans le monde de barrages gonflables sur la crête d'un barrage voûte. Le barrage de Pit Three, en Californie septentrionale, est exploité par le Maître de l'Ouvrage, la Pacific Gas and Electric Company, la plus grande société privée de services du monde. Les trois portées de $1,83 \mathrm{~m}$ de haut et 25,53 m de long augmentent la hauteur de chute d'eau pour l'usine de 89 MW tout en augmentant la capacité de la retenue, une fonction importante dans les conditions arides de la Californie.

Les trois barrages gonflables remplacent les hausses de déversoir. Une des conditions exigées étant que les hausses soient capables de laisser passer les eaux de crue alors même qu'elles restaient en place obligeait à réduire leur hauteur à la moitié de celle possible avec un système à ouverture automatique. Par contre, l'installation de clapets conventionnels sur la crête de la voûte impliquait non seulement un coût excessif, mais aussi des difficultés techniques considérables à cause du rayon de courbure de la voûte de $152,5 \mathrm{~m}$. Les barrages gonflables constituaient la solution intermédiaire entre ces deux approches.

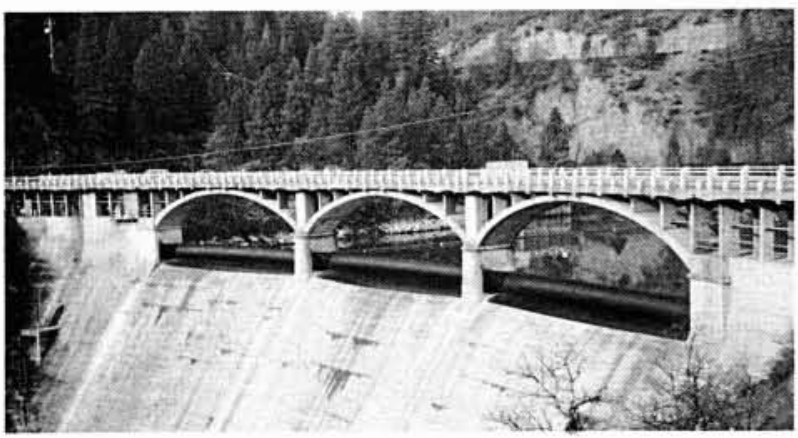

